\title{
Editorial
}

\section{From the Editors: Introducing a New Editing Team and Welcoming a New Year}

\author{
Anne S. Tsui ${ }^{1,2}$ and Kwok Leung ${ }^{3}$ \\ 'Arizona State University, USA, ${ }^{2}$ Peking University, China, and ${ }^{3}$ City University of Hong Kong, \\ China
}

Volume seven of Management and Organization Review brings to our readers some exciting news. We are proud to introduce newly reconfigured Editing, Review, and Advisory Boards of $M O R$.

\section{THANK YOU AND WELCOME TO MEMBERS OF THE EDITING, REVIEW, AND ADVISORY BOARDS}

We are pleased to announce that Kwok Leung has assumed the position of Deputy Editor-in-Chief, and will work in partnership with Anne Tsui to launch the next stage of development of MOR. Kwok is a Founding Senior Editor of $M O R$. His past editorial service includes being a Departmental Editor (20022005) and a Deputy Editor-in-Chief (2006-2007) of the Journal of International Business Studies, the Editor of the Asia Fournal of Social Psychology (2000-2005), and an Associate Editor of Asia Pacific Journal of Management (2001-2003). Kwok's extensive experience along with his commitment to Chinese management and cross-cultural research will elevate $M O R$ 's development as a leading journal for insights on both management within the Chinese context and East-West comparative and integrative research.

A second change is that Marshall W. Meyer will assume the title of Executive Senior Editor focusing on relations with publishers and universities, and ensuring the long-term sustainability of the journal. Marshall is also a Founding Senior Editor of MOR, was Associate Editor of Administrative Science Quarterly (1987-1995), and has served on many editorial and review boards.

Senior Editor Jiing-Lih Farh and Anne Tsui gave birth to the idea of the journal at the HKUST office. Marshall Meyer and Anne Tsui subsequently solidified it 
over a glass of wine in a Lan Gui Fong bar in mid level Hong Kong. Every Founding Editor has played a unique role for the journal and we thank them for their continuing support and guidance.

Among the editors, Yanjie Bian, John Child, and Doug Guthrie, Founding Senior Editors, and Shuming Zhao, Consulting Editor, will be joining the Editorial Advisory Board. We thank them for their 7 years of service as editors and will appreciate their continuing contribution as advisors to further advance $M O R$. We also thank the Editorial Advisory Board members who have decided to step down: George Milkovich and Richard Mowday. We call upon the advisors for ideas to develop the journal and to serve as action editors on particular manuscripts that can benefit especially from their expertise. We thank them for their generous support and continuing commitment to the development of $M O R$ that publishes high quality and important work with relevance for practice for firms in China and beyond.

Joining the Editing team will be Zhi-Xue Zhang and Chung-Ming Lau as Senior Editors, and Ingmar Bjorkman, Chao Chen, and George Zhen Xiong Chen as Consulting Editors. The five new editors come from four countries (Australia, China, Finland, and the U.S.) and four continents. The entire Editing team is comprised of 18 editors evenly distributed for macro (firm level analysis) and micro topics (individual and team level). This team of editors commits to serving the authors and readers of $M O R$ with a highly developmental philosophy to select and nurture promising papers that provide great insight on unique and general management phenomena in the Chinese context and beyond.

To support the editors is an outstanding international Editorial Review Board with 38 continuing members and 22 new members. The Board of 60 reviewers consists of scholars from 49 universities in 12 countries/regions, covering four continents. We welcome the following new members to the Editorial Review Board: Bat Batjargal, Wenhong Chen, Chi-Nien Chung, Ping Deng, Ray Friedman, Barry Gerhart, Yaping Gong, Paul S. Hempel, Yingyi Hong, Liangding Jia, Sunghoon Kim, Jing Li, Zhiang (John) Lin, Jane Lu, Jiangyong Lu, Lin Lu, Mona Makhija, Kok-yee Ng, Lynda Jiwen Song, Hinrich Voss, Jianjun Zhang, and Yingying Zhang. MOR could not have achieved our reputation for being a journal where authors receive highly constructive and developmental feedback were it not for the high quality, constructive, and developmental reviews written by our reviewers. We thank the 14 editorial review board members who have completed their service for their valuable contribution in the founding years of MOR. They are: David Ahlstrom, Winton Au, Yang Cao, Andrew Delios, Gregory Dess, Charles Dhanaraj, Christopher Hsee, Gary Latham, Klaus Meyer, Keith Murnighan, Mike Peng, Yijiang Wang, Elke Weber, and Aimin Yan.

Reviewing is both a responsibility in our profession and it is also a highly volitional activity (Hames, 2007; Trevino, 2008; Tsui \& Hollenbeck, 2009). 
Reviewers give up part of their valuable time to support, anonymously, the development of papers written by colleagues whom they have not met and may never meet. Their reviews provide important and necessary input to help the editors in making informed and fair decisions about the manuscripts while providing valuable suggestions to the authors to further improve their work whether this work will or will not be published in MOR. The reviewers are unknown to the authors and they may never receive a word of praise or thanks from the authors. These behind-the-scenes contributions are both altruistic and instrumental. They are altruistic because each scholar can choose to make this contribution without explicit rewards. They are instrumental because peer review is the only mechanism we have defined in our field as the legitimate way to identify research that meets the scientific standard of rigor in conceptual analysis and empirical methods. Reviewing also helps the development of a field because the goal of reviewing, especially at $M O R$, is not only to evaluate and select those works that are worthy of being shared in the public domain, but also to help develop the field through its developmental and instructive comments to authors. We are grateful to our reviewers for their willingness to contribute to these noble goals.

\section{REFERENCES}

Hames, I. 2007. Peer revieze and manuscript management in scientific journals. Malden, MA: Blackwell.

Trevino, L. K. 2008. Why review? Because reviewing is a professional responsibility. Academy of Management Revieze, 33(1): 8-10.

Tsui, A. S., \& Hollenbeck, J. R. 2009. Successful authors and effective reviewers: Balancing supply and demand in organizational sciences. Organizational Research Methods, 12(2): 259272.

\section{THE YEAR OF THE RABBIT}

The cover image of this issue, titled 'Jade Rabbit Making Heavenly Medicine' by artist Lanying Hao, is to commemorate the Chinese New Year, which is the year of the Rabbit. The rabbit is a very popular symbolic image in Chinese culture. It means gentleness and wisdom.

According to Ms. Hao, the paper-cut means the God of Rabbit is making immortal medicine for kind people and blessing them with prosperity. What is the medicine for the Chinese Capital Market? What can give it everlasting prosperity?

Lanying Hao is a peasant from the outskirts of Beijing and a member of the China Association of Folk Literature and Art as a paper-cut artist. She is well known as 'the most nimble wife' in China. She is so skilful that the flowers, fish, and birds that she cut are as vivid as alive. Nobody would believe that she made them just with a small pair of scissors. Her paper-cutting works have won a number of awards in China and have been exhibited in galleries and museums. 